Also available at http://amc-journal.eu

ISSN 1855-3966 (printed edn.), ISSN 1855-3974 (electronic edn.)

ARS MATHEMATICA CONTEMPORANEA 13 (2017) 227-234

\title{
Counting faces of graphical zonotopes
}

\author{
Vladimir Grujić * \\ University of Belgrade, Faculty of Mathematics, \\ Studentski trg 16, Belgrade, Serbia
}

Received 15 June 2016, accepted 16 January 2017, published online 6 March 2017

\begin{abstract}
It is a classical fact that the number of vertices of the graphical zonotope $Z_{\Gamma}$ is equal to the number of acyclic orientations of a graph $\Gamma$. We show that the $f$-polynomial of $Z_{\Gamma}$ is obtained as the principal specialization of the $q$-analog of the chromatic symmetric function of $\Gamma$.
\end{abstract}

Keywords: Graphical zonotope, f-vector, graphical matroid, symmetric function.

Math. Subj. Class.: 05E05, 52B05, 16705

\section{Introduction}

The $f$-polynomial of an $n$-dimensional polytope $P$ is defined by $f(P, q)=\sum_{i=0}^{n} f_{i}(P) q^{i}$, where $f_{i}(P)$ is the number of $i$-dimensional faces of $P$. The $f$-polynomial $f\left(\mathcal{Z}_{\Gamma}, q\right)$ of the graphical zonotope $\mathcal{Z}_{\Gamma}$ is a combinatorial invariant of a finite, simple graph $\Gamma$. The vertices of $\mathcal{Z}_{\Gamma}$ are in one-to-one correspondence with regions of the graphical hyperplane arrangement $\mathcal{H}_{\Gamma}$, which are enumerated by acyclic orientations of $\Gamma$.

Stanley's chromatic symmetric function $\Psi(\Gamma)=\sum_{f \text { proper }} \mathbf{x}_{f}$ of a graph $\Gamma=(V, E)$, introduced in [7], is the enumerator function of proper colorings $f: V \rightarrow \mathbb{N}$, where $\mathbf{x}_{f}=x_{f(1)} \cdots x_{f(n)}$ and $f$ is proper if there are no monochromatic edges. The chromatic polynomial $\chi(\Gamma, d)$ of the graph $\Gamma$, which counts proper colorings with a finite number of colors, appears as the principal specialization

$$
\chi(\Gamma, d)=\operatorname{ps}(\Psi(\Gamma))(d)=\left.\Psi(\Gamma)\right|_{x_{1}=\cdots=x_{d}=1, x_{d+1}=\cdots=0} .
$$

The number of acyclic orientations of $\Gamma$ is determined by the value of the chromatic polynomial $\chi(\Gamma, d)$ at $d=-1,[6]$

$$
a(\Gamma)=(-1)^{|V|} \chi(\Gamma,-1) .
$$

* Author is supported by Ministry of Education, Science and Technological developments of Republic of Serbia, Project 174034.

E-mail address: vgrujic@matf.bg.ac.rs (Vladimir Grujić)

(a) (i) This work is licensed under http://creativecommons.org/licenses/by/3.0/ 
There is a $q$-analog of the chromatic symmetric function $\Psi_{q}(\Gamma)$ introduced in a wider context of the combinatorial Hopf algebra of simplicial complexes considered in [2]. It is a symmetric function over the field of rational functions in $q$. The principal specialization of $\Psi_{q}(\Gamma)$ is the $q$-analog of the chromatic polynomial $\chi_{q}(\Gamma, d)$.

The main result of this paper is the following generalization of formula (1.1):

Theorem 1.1. Let $\Gamma=(V, E)$ be a simple connected graph and $\mathcal{Z}_{\Gamma}$ the corresponding graphical zonotope. Then the $f$-polynomial of $\mathcal{Z}_{\Gamma}$ is given by

$$
f\left(\mathcal{Z}_{\Gamma}, q\right)=(-1)^{|V|} \chi_{-q}(\Gamma,-1) .
$$

The cancellation-free formula for the antipode in the Hopf algebra of graphs, obtained by Humpert and Martin in [3], reflects the fact that $f\left(\mathcal{Z}_{\Gamma}, q\right)$ depends only on the graphical matroid $M(\Gamma)$ associated to $\Gamma$. For instance, for any tree $T_{n}$ the graphical matroid is the uniform matroid $M\left(T_{n}\right)=U_{n}^{n}$ and the corresponding graphical zonotope is the cube $\mathcal{Z}_{T_{n}}=I^{n-1}$. Whitney's theorem from 1933 describes how two graphs with the same graphical matroid are related [9]. It can be used to find more interesting nonisomorphic graphs with the same $f$-polynomials of corresponding graphical zonotopes.

The paper is organized as follows. In Section 2, we review the basic facts about zonotopes. In Section 3, the $q$-analog of the chromatic symmetric function $\Psi_{q}(\Gamma)$ of a graph $\Gamma$ is introduced. Theorem 1.1 is proved in Section 4. We present some examples and calculations in Section 5.

\section{Zonotopes}

A zonotope $\mathcal{Z}=\mathcal{Z}\left(v_{1}, \ldots, v_{m}\right)$ is a convex polytope determined by a collection of vectors $\left\{v_{1}, \ldots, v_{m}\right\}$ in $\mathbb{R}^{n}$ as the Minkowski sum of line segments

$$
\mathcal{Z}=\left[-v_{1}, v_{1}\right]+\cdots+\left[-v_{m}, v_{m}\right]
$$

It is a projection of the $m$-cube $[-1,1]^{m}$ under the linear map $\mathbf{t} \mapsto A \mathbf{t}, \mathbf{t} \in[-1,1]^{m}$, where $A=\left[v_{1} \cdots v_{m}\right]$ is an $n \times m$-matrix whose columns are vectors $v_{1}, \ldots, v_{m}$. The zonotope $\mathcal{Z}$ is symmetric about the origin and all its faces are translations of zonotopes.

To a collection of vectors $\left\{v_{1}, \ldots, v_{m}\right\}$ is associated a central arrangement of hyperplanes $\mathcal{H}=\left\{H_{v_{1}}, \ldots, H_{v_{m}}\right\}$, where $H_{v}$ denotes the hyperplane perpendicular to a vector $v \in \mathbb{R}^{n}$. The zonotope $\mathcal{Z}$ and the corresponding arrangement of hyperplanes $\mathcal{H}$ are closely related. In fact the associated fan $\mathcal{F}_{\mathcal{H}}$ of the arrangement $\mathcal{H}$ is the normal fan $\mathcal{N}(\mathcal{Z})$ of the zonotope $\mathcal{Z}$ (see [10, Theorem 7.16]). It follows that the face lattice of $\mathcal{F}_{\mathcal{H}}$ and the reverse face lattice of $\mathcal{Z}$ are isomorphic. In particular, vertices of $\mathcal{Z}$ correspond to regions of $\mathcal{H}$ and their total numbers coincide

$$
f_{0}(\mathcal{Z})=r(\mathcal{H})
$$

The faces of the zonotope $\mathcal{Z}$ are encoded by covectors of the oriented matroid $\mathcal{M}$ associated to the collection of vectors $\left\{v_{1}, \ldots, v_{m}\right\}$. The covectors are sign vectors

$$
\mathcal{V}^{*}=\left\{\operatorname{sign}(v) \in\{+,-, 0\}^{m} \mid v \in \mathbb{R}^{n}\right\},
$$

where $\operatorname{sign}(v)_{i}=\left\{\begin{array}{cc}+, & \left\langle v, v_{i}\right\rangle>0 \\ 0, & \left\langle v, v_{i}\right\rangle=0 \\ -, & \left\langle v, v_{i}\right\rangle<0\end{array}, i=1, \ldots, m\right.$. The face lattice of the zonotope $\mathcal{Z}$ is isomorphic to the lattice of covectors componentwise induced by,$+-<0$ on $\mathcal{V}^{*}$. 
A special class of zonotopes is determined by simple graphs. To a connected graph $\Gamma=(V, E)$, whose vertices are enumerated by integers $V=\{1, \ldots, n\}$, are associated the graphical zonotope

$$
\mathcal{Z}_{\Gamma}=\mathcal{Z}\left(e_{i}-e_{j} \mid i<j,\{i, j\} \in E\right)
$$

and the graphical arrangement in $\mathbb{R}^{n}$

$$
\mathcal{H}_{\Gamma}=\left\{H_{e_{i}-e_{j}} \mid i<j,\{i, j\} \in E\right\}
$$

There is a bijective correspondence between regions of $\mathcal{H}_{\Gamma}$ and acyclic orientations of $\Gamma$, [8, Proposition 2.5], which by (2.1) implies

$$
f_{0}\left(\mathcal{Z}_{\Gamma}\right)=r\left(\mathcal{H}_{\Gamma}\right)=a(\Gamma)
$$

The arrangement $\mathcal{H}_{\Gamma}$ is refined by the braid arrangement $\mathcal{A}_{n-1}$ consisting of all hyperplanes $H_{e_{i}-e_{j}}, 1 \leq i<j \leq n$. Thus $\mathcal{Z}_{\Gamma}$ belongs to a wider class of convex polytopes called generalized permutohedra introduced in [4]. Since arrangements $\mathcal{H}_{\Gamma}$ and $\mathcal{A}_{n-1}$ are not essential we take their quotients by the line $l: x_{1}=\cdots=x_{n}$ and without confusing retain the same notation. Consequently $\operatorname{dim} \mathcal{Z}_{\Gamma}=n-1$.
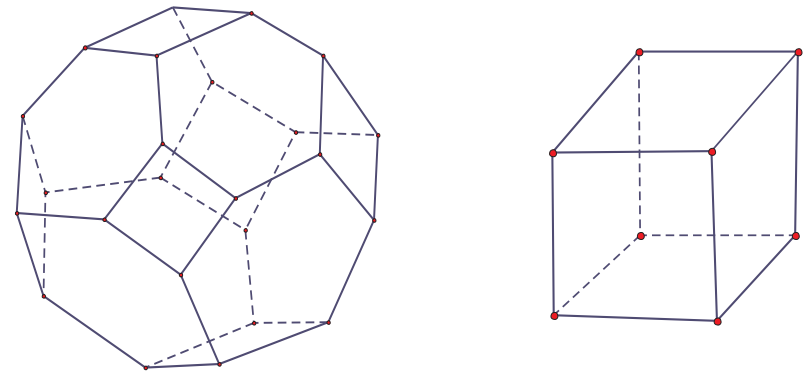

Figure 1: Permutohedron $P e^{3}$ and cube $I^{3}$.

Example 2.1. (i) The permutohedron $P e^{n-1}$ is represented as the graphical zonotope $\mathcal{Z}_{K_{n}}$ corresponding to the complete graph $K_{n}$ on $n$ vertices (Figure 1).

(ii) The cube $I^{n-1}$ is represented as the graphical zonotope $\mathcal{Z}_{T_{n}}$ corresponding to an arbitrary tree $T_{n}$ on $n$ vertices. This shows that the graph $\Gamma$ is not determined by the combinatorial type of the zonotope $\mathcal{Z}_{\Gamma}$.

\section{$3 q$-analog of chromatic symmetric function of graph}

Stanley's chromatic symmetric function $\Psi(\Gamma)$ can be obtained in a purely algebraic way. A combinatorial Hopf algebra $\mathcal{H}$ is a graded, connected Hopf algebra equipped with the multiplicative linear functional $\zeta: \mathcal{H} \rightarrow \mathbf{k}$ to the ground field $\mathbf{k}$. For the theory of combinatorial Hopf algebras see [1]. Consider the combinatorial Hopf algebra of graphs $\mathcal{G}$ which is linearly generated over a field $\mathbf{k}$ by simple finite graphs with the product defined by disjoint union $\Gamma_{1} \cdot \Gamma_{2}=\Gamma_{1} \sqcup \Gamma_{2}$ and the coproduct

$$
\Delta(\Gamma)=\left.\left.\sum_{I \subset V} \Gamma\right|_{I} \otimes \Gamma\right|_{V \backslash I}
$$


where $\left.\Gamma\right|_{I}$ denotes the induced subgraph on $I \subset V$. The structure of $\mathcal{G}$ is completed by the character $\zeta: \mathcal{G} \rightarrow \mathbf{k}$ defined to be $\zeta(\Gamma)=1$ for $\Gamma$ with no edges and $\zeta(\Gamma)=0$ otherwise. Then it turns out that $\Psi(\Gamma)$ is the image of the unique morphism of combinatorial Hopf algebras to symmetric functions $\Psi: \mathcal{G} \rightarrow$ Sym, ([1, Example 4.5]).

An important part of the structure of the Hopf algebra $\mathcal{G}$ is the antipode $S: \mathcal{G} \rightarrow \mathcal{G}$. The cancellation-free formula for the antipode in terms of acyclic orientations of a graph $\Gamma$ is obtained in [3]. We recall some basic definitions. Terminology comes from matroid theory. Given a graph $\Gamma=(V, E)$, for a collection of edges $F \subset E$ denote by $\Gamma_{V, F}$ the graph on $V$ with the edge set $F$. A flat $F$ of the graph $\Gamma$ is a collection of its edges such that components of $\Gamma_{V, F}$ are induced subgraphs. The rank $\operatorname{rk}(F)$ is the size of spanning forests of $\Gamma_{V, F}$. We have that $|V|=\operatorname{rk}(F)+c(F)$, where $c(F)$ is the number of components of $\Gamma_{V, F}$. By contracting edges from a flat $F$ we obtain the graph $\Gamma / F$. Finally, let $a(\Gamma)$ be the number of acyclic orientations of $\Gamma$. The formula of Humpert and Martin is as follows

$$
S(\Gamma)=\sum_{F \in \mathcal{F}(\Gamma)}(-1)^{c(F)} a(\Gamma / F) \Gamma_{V, F},
$$

where the sum is over the set of flats $\mathcal{F}(\Gamma)$.

The following modification of the character $\zeta$ is considered in [2] in a wider context of the combinatorial Hopf algebra of simplicial complexes. Define $\zeta_{q}(\Gamma)=q^{\mathrm{rk}(\Gamma)}$, which determines the algebra morphism $\zeta_{q}: \mathcal{G} \rightarrow \mathbf{k}(q)$, where $\mathbf{k}(q)$ is the field of rational functions in $q$. This character produces the unique morphism $\Psi_{q}: \mathcal{G} \rightarrow$ QSym to quasisymmetric functions over $\mathbf{k}(q)$. The expansion of $\Psi_{q}(\Gamma)$ in the monomial basis of quasisymmetric functions is determined by the universal formula [1, Theorem 4.1]

$$
\Psi_{q}(\Gamma)=\sum_{\alpha \models n}\left(\zeta_{q}\right)_{\alpha}(\Gamma) M_{\alpha}
$$

The sum above is over all compositions of the integer $n=|V|$ and the coefficient of the expansion corresponding to the composition $\alpha=\left(a_{1}, \ldots, a_{k}\right) \models n$ is given by

$$
\left(\zeta_{q}\right)_{\alpha}(\Gamma)=\sum_{I_{1} \sqcup \ldots \sqcup I_{k}=V} q^{\mathrm{rk}\left(\left.\Gamma\right|_{I_{1}}\right)+\cdots+\operatorname{rk}\left(\left.\Gamma\right|_{I_{k}}\right)},
$$

where the sum is over all set compositions of $V$ of the type $\alpha$. The coefficients $\left(\zeta_{q}\right)_{\alpha}(\Gamma)$ depend only on the partition corresponding to a composition $\alpha$, so the function $\Psi_{q}(\Gamma)$ is actually symmetric and it can be expressed in the monomial basis of symmetric functions.

The invariant $\Psi_{q}(\Gamma)$ is more subtle than $\Psi(\Gamma)$. Obviously $\Psi_{0}(\Gamma)$ is the chromatic symmetric function of a graph $\Gamma$. It remains open to find two nonisomorphic graphs $\Gamma_{1}$ and $\Gamma_{2}$ with the same $q$-chromatic symmetric functions $\Psi_{q}\left(\Gamma_{1}\right)=\Psi_{q}\left(\Gamma_{2}\right)$. Let

$$
\chi_{q}(\Gamma, d)=\operatorname{ps}\left(\Psi_{q}(\Gamma)\right)(d)
$$

be the $q$-analog of the chromatic polynomial $\chi(\Gamma, d)$. It is a consequence of a general fact for combinatorial Hopf algebras (see [1]) that

$$
\chi_{q}(\Gamma,-1)=\left(\zeta_{q} \circ S\right)(\Gamma)
$$


Example 3.1. Consider the graph $\Gamma$ on four vertices with the edge set $E=\{12,13,23,34\}$. We find that

$$
\Psi_{q}(\Gamma)=24 m_{1,1,1,1}+(8 q+4) m_{2,1,1}+\left(2 q^{2}+4 q\right) m_{2,2}+\left(3 q^{2}+q\right) m_{3,1}+q^{3} m_{4} .
$$

By principal specialization and taking into account that

$$
\operatorname{ps}\left(m_{\lambda_{1}^{i_{1}}, \ldots, \lambda_{k}^{i_{k}}}\right)(d)=\frac{\left(i_{1}+\cdots+i_{k}\right) !}{i_{1} ! \cdots i_{k} !}\left(\begin{array}{c}
d \\
i_{1}+\cdots+i_{k}
\end{array}\right),
$$

we obtain

$$
\chi_{q}(\Gamma, d)=d(d-1)^{2}(d-2)+q d(d-1)(4 d-5)+4 q^{2} d(d-1)+q^{3} d,
$$

which by Theorem 1.1 gives

$$
f\left(\mathcal{Z}_{\Gamma}, q\right)=12+18 q+8 q^{2}+q^{3} .
$$

\section{Proof of Theorem 1.1}

Proof. By applying (3.2) and the formula for antipode (3.1) we obtain

$$
(-1)^{|V|} \chi_{-q}(\Gamma,-1)=(-1)^{|V|} \sum_{F \in \mathcal{F}(\Gamma)}(-1)^{c(\Gamma)} a(\Gamma / F)(-q)^{\operatorname{rk}(F)} .
$$

It follows that the statement of the theorem is equivalent to the following expression of the $f$-polynomial

$$
f\left(\mathcal{Z}_{\Gamma}, q\right)=\sum_{F \in \mathcal{F}(\Gamma)} a(\Gamma / F) q^{\mathrm{rk}(F)} .
$$

Therefore it should be shown that components of $f$-vectors are determined by

$$
f_{k}\left(\mathcal{Z}_{\Gamma}\right)=\sum_{\substack{F \in \mathcal{F}(\Gamma) \\ \operatorname{rk}(F)=k}} a(\Gamma / F), 0 \leq k \leq n-1 .
$$

By duality between the face lattice of $\mathcal{Z}_{\Gamma}$ and the face lattice of the fan $\mathcal{F}_{\mathcal{H}_{\Gamma}}$ we have

$$
f_{k}\left(\mathcal{Z}_{\Gamma}\right)=f_{n-k-1}\left(\mathcal{F}_{\mathcal{H}_{\Gamma}}\right)
$$

Let $L\left(\mathcal{H}_{\Gamma}\right)$ be the intersection lattice of the graphical arrangement $\mathcal{H}_{\Gamma}$. For a subspace $X \in L\left(\mathcal{H}_{\Gamma}\right)$ there is an arrangement of hyperplanes

$$
\mathcal{H}_{\Gamma}^{X}=\left\{X \cap H \mid X \nsubseteq H, H \in \mathcal{H}_{\Gamma}\right\}
$$

whose intersection lattice $L\left(\mathcal{H}_{\Gamma}^{X}\right)$ is isomorphic to the upper cone of $X$ in $L\left(\mathcal{H}_{\Gamma}\right)$. Since $\mathcal{H}_{\Gamma}$ is central and essential we have

$$
f_{n-k-1}\left(\mathcal{F}_{\mathcal{H}_{\Gamma}}\right)=\sum_{\substack{X \in L\left(\mathcal{H}_{\Gamma}\right) \\ \operatorname{dim}(X)=n-k-1}} r\left(\mathcal{H}_{\Gamma}^{X}\right)
$$

where $r\left(\mathcal{H}_{\Gamma}^{X}\right)$ is the number of regions of the arrangement $\mathcal{H}_{\Gamma}^{X}$, see [8, Theorem 2.6].

The intersection lattice $L\left(\mathcal{H}_{\Gamma}\right)$ is isomorphic to the lattice of flats of the graphical matroid $M(\Gamma)$. By this isomorphism to a flat $F$ of rank $k$ corresponds the intersection subspace $X^{F}=\cap_{\{i, j\} \in F} H_{e_{i}-e_{j}}$ of dimension $n-k-1$. It is easy to see that arrangements $\mathcal{H}_{\Gamma}^{X^{F}}$ and $\mathcal{H}_{\Gamma / F}$ coincide, which by (2.2) and comparing formulas (4.2) and (4.3) proves theorem. 


\section{Examples}

By applying Theorem 1.1 we obtain the following interpretation of identities elaborated in [2, Propositions 17, 19].

Example 5.1. (i) For the permutohedron $P e^{n-1}=\mathcal{Z}_{K_{n}}$, the $f$-polynomial is given by

$$
f\left(\mathcal{Z}_{K_{n}}, q\right)=A_{n}(q+1)
$$

where $A_{n}(q)=\sum_{\pi \in S_{n}} q^{\operatorname{des}(\pi)}$ is the Euler polynomial. Recall that $\operatorname{des}(\pi)$ is the number of descents of a permutation $\pi \in S_{n}$. It recovers the fact that the $h$-polynomial of the permutohedron $P e^{n-1}$ is the Euler polynomial $A_{n}(q)$.

(ii) For the cube $I^{n-1}=\mathcal{Z}_{T_{n}}$, where $T_{n}$ is a tree on $n$ vertices, the $f$-polynomial is given by

$$
f\left(\mathcal{Z}_{T_{n}}, q\right)=(q+2)^{n-1}
$$

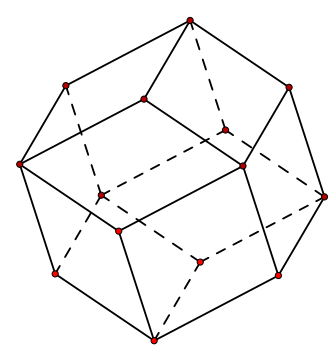

Figure 2: Rhombic dodecahedron $\mathcal{Z}_{C_{4}}$.

Proposition 5.2. The $f$-polynomial of the graphical zonotope $\mathcal{Z}_{C_{n}}$ associated to the cycle graph $C_{n}$ on $n$ vertices is given by

$$
f\left(\mathcal{Z}_{C_{n}}, q\right)=q^{n}+q^{n-1}+(q+2)^{n}-2(q+1)^{n} .
$$

Proof. A flat $F \in \mathcal{F}\left(C_{n}\right)$ is determined by the complementary set of edges. If $\operatorname{rk}(F)=$ $n-k, k>1$ then the complementary set has $k$ edges and $C_{n} / F=C_{k}$. Since $a\left(C_{k}\right)=$ $2^{k}-2, k>1$, by formula (4.2), we obtain

$$
f_{n-k}\left(\mathcal{Z}_{C_{n}}\right)=\left(2^{k}-2\right)\left(\begin{array}{l}
n \\
k
\end{array}\right), 2 \leq k \leq n,
$$

which leads to the required formula.

Specially, for $n=4$ the resulting zonotope is the rhombic dodecahedron (see Figure 2). We have

$$
f\left(\mathcal{Z}_{C_{4}}, q\right)=14+24 q+12 q^{2}+q^{3} .
$$

Proposition 5.3. Let $\Gamma=\Gamma_{1} \vee_{v} \Gamma_{2}$ be the wedge of two connected graphs $\Gamma_{1}$ and $\Gamma_{2}$ at the common vertex $v$. Then

$$
f\left(\mathcal{Z}_{\Gamma}, q\right)=f\left(\mathcal{Z}_{\Gamma_{1}}, q\right) f\left(\mathcal{Z}_{\Gamma_{2}}, q\right)
$$


Proof. The graphical matroids of involving graphs are related by $M(\Gamma)=M\left(\Gamma_{1}\right) \oplus$ $M\left(\Gamma_{2}\right)$. For the sets of flats it holds $\mathcal{F}(\Gamma)=\left\{F_{1} \cup F_{2} \mid F_{i} \in \mathcal{F}\left(\Gamma_{i}\right), i=1,2\right\}$. For $F=F_{1} \cup F_{2}$ we have $\Gamma / F=\Gamma_{1} / F_{1} \vee_{[v]} \Gamma_{2} / F_{2}$, where $[v]$ is the component of the vertex $v$ in $\Gamma_{V, F}$. Obviously $a(\Gamma / F)=a\left(\Gamma_{1} / F_{1}\right) a\left(\Gamma_{2} / F_{2}\right)$ and $\operatorname{rk}(F)=\operatorname{rk}\left(F_{1}\right)+\operatorname{rk}\left(F_{2}\right)$. The proposition follows from formula (4.1).

The formula for cubes in Example 5.1 (ii) follows from Proposition 5.3 since any tree is a consecutive wedge of edges and $f\left(I^{1}, q\right)=q+2$. It also allows us to restrict ourselves only to biconnected graphs. For a biconnected graph $\Gamma$ with a disconnecting pair of vertices $\{u, v\}$ Whitney introduced the transformation called the $t$ wist around the pair $\{u, v\}$. This transformation does not have an affect on the graphical matroid $M(\Gamma)$ [9].

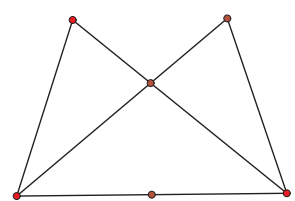

$\Gamma_{1}$

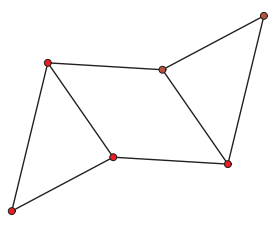

$\Gamma_{2}$

Figure 3: Biconnected graphs related by twist transformation.

Example 5.4. Figure 3 shows the pair of biconnected graphs on six vertices obtained one from another by the twist transformation. The corresponding zonotopes have the same $f$-polynomial

$$
f\left(\mathcal{Z}_{\Gamma_{1}}, q\right)=f\left(\mathcal{Z}_{\Gamma_{2}}, q\right)=126+348 q+358 q^{2}+164 q^{3}+30 q^{4}+q^{5} .
$$

On the other hand their $q$-chromatic symmetric functions are different. One can check that corresponding coefficients by $m_{3,1^{3}}$ are different

$$
\begin{aligned}
& {\left[m_{3,1^{3}}\right] \Psi_{q}\left(\Gamma_{1}\right)=\left(11 q^{2}+8 q+1\right) \cdot 3 !} \\
& {\left[m_{3,1^{3}}\right] \Psi_{q}\left(\Gamma_{2}\right)=\left(10 q^{2}+10 q\right) \cdot 3 !}
\end{aligned}
$$

This shows that the $q$-analog of the chromatic symmetric function of a graph is not determined by the corresponding graphical matroid. By taking $q=0$ we obtain that even the chromatic symmetric functions are different since $\left[m_{3,1^{3}}\right] \Psi\left(\Gamma_{1}\right)=6$ and $\left[m_{3,1^{3}}\right] \Psi\left(\Gamma_{2}\right)=$ 0 .

Let us now consider Stanley's example of nonisomorphic graphs with the same chromatic symmetric functions, see [7]. We find that the $f$-polynomials of the corresponding graphical zonotopes differ for those graphs. From these examples we conclude that chromatic properties of a graph and the $f$-vector of the corresponding graphical zonotope are not related.

We have already noted that graphical zonotopes are generalized permutohedra. The $h$-polynomials of simple generalized permutohedra are determined in [5, Theorem 4.2]. The only simple graphical zonotopes are products of permutohedra [5, Proposition 5.2]. They are characterized by graphs whose biconnected components are complete subgraphs. Therefore Proposition 5.3 together with Example 5.1 (i) prove that the $h$-polynomial of a 
simple graphical zonotope is the product of Eulerian polynomials, the fact obtained in [5, Corollary 5.4]. Example 3.1 is of this sort and represents the hexagonal prism which is the product $\mathcal{Z}_{K_{3}} \times \mathcal{Z}_{K_{2}}$.

\section{References}

[1] M. Aguiar, N. Bergeron and F. Sottile, Combinatorial Hopf algebras and generalized DehnSommerville relations, Compos. Math. 142 (2006), 1-30, doi:10.1112/s0010437x0500165x.

[2] C. Benedetti, J. Hallam and J. Machacek, Combinatorial Hopf algebras of simplicial complexes, SIAM J. Discrete Math. 30 (2016), 1737-1757, doi:10.1137/15m1038281.

[3] B. Humpert and J. L. Martin, The incidence Hopf algebra of graphs, SIAM J. Discrete Math. 26 (2012), 555-570, doi:10.1137/110820075.

[4] A. Postnikov, Permutohedra, associahedra, and beyond, Int. Math. Res. Not. 2009 (2009), 1026-1106, doi:10.1093/imrn/rnn153.

[5] A. Postnikov, V. Reiner and L. Williams, Faces of generalized permutohedra, Doc. Math. 13 (2008), 207-273, http://www . math. uiuc . edu/documenta/vol-13/10.html.

[6] R. P. Stanley, Acyclic orientations of graphs, Discrete Math. 5 (1973), 171-178, doi:10.1016/ 0012-365x(73)90108-8.

[7] R. P. Stanley, A symmetric function generalization of the chromatic polynomial of a graph, Adv. Math. 111 (1995), 166-194, doi:10.1006/aima.1995.1020.

[8] R. P. Stanley, An introduction to hyperplane arrangements, in: E. Miller, V. Reiner and B. Sturmfels (eds.), Geometric Combinatorics, American Mathematical Society, Providence, Rhode Island, volume 13 of IAS/Park City Mathematics Series, pp. 389-496, 2007.

[9] H. Whitney, 2-isomorphic graphs, Amer. J. Math. 55 (1933), 245-254, doi:10.2307/2371127.

[10] G. M. Ziegler, Lectures on Polytopes, volume 152 of Graduate Texts in Mathematics, SpringerVerlag, New York, 1995, doi:10.1007/978-1-4613-8431-1. 